\title{
Improved Recombinant Retroviral Titers Utilizing Trichostatin A
}

BioTechniques 29:884-890(October 2000)

\author{
Chris A. Tobias, Duckhyun \\ Kim and Itzhak Fischer \\ MCP Hahnemann University, \\ Philadelphia, PA, USA
}

\section{INTRODUCTION}

Gene therapy is a powerful tool for correcting genetic and neurodegenerative disorders, and provides a means for therapeutic interventions after traumatic injury. One aspect of gene therapy is ex vivo gene therapy, which involves the genetic modification of cells that will be introduced back into a host to provide a therapeutic gene that will aid in recovery. The advantage of using recombinant retroviruses for ex vivo gene therapy is that they integrate into the genome of the cells infected, resulting in the stable transfer of the gene to the daughter cells. The main disadvantage of recombinant retroviruses is their low viral titers, which limits the number of cells infected by the virus and subsequently their efficiency upon transplantation into the host.

Several strategies have been used to increase the usefulness of retroviruses by increasing the viral titer. One com mon method is to centrifuge large batches of producer cell supernatant to concentrate the virus for infection (16). This method can increase the number of viral particles in a retroviral infection, but large amounts of the virus can be lost in the harvesting and centrifuging because the virus is labile (10). This method therefore results in concentrated, yet inconsistent viral stocks. Lowering the temperature during the production and harvesting of viral supernatant from producer cell lines has been shown to increase the viral titer up to tenfold $(7,8)$. Using low concentrations of essential growth-promoting proteins such as serum during retroviral harvest can also increase the titer of a retrovirus several fold (5).

One of the most useful methods for increasing retroviral titers has been the application of the drug sodium butyrate during the production, harvesting and infection with a retrovirus. Sodium butyrate is a known inhibitor of the histone deacetylases (1). Although it may have other actions in a cell (9), sodium butyrate has been consistently found to increase the viral titer in a number of different amphotropic producer cells (15). The increase in viral titer depends on several factors, including the producer cell line, the viral construct and the cell line that was infected (15).

We investigated the application of other drugs that can further improve the retrovirus titer. The microbial metabolite, trichostatin A, is a specific inhibitor of histone deacetylase $(21,22)$ and has previously been shown to be a more potent inhibitor of histone deacetylase than sodium butyrate at micromolar concentrations (2). In addition, trichostatin A has been reported to have the ability to reactivate silenced genes in cell lines transduced by an adeno-associated virus (AAV) (2) or a baculovirus (4). In this report, we treated an ecotropic producer cell line ( $\left.y^{2} / \mathrm{LIG} / \mathrm{NGF}\right)$ with a range of concentrations of trichostatin A to determine if it would increase the viral titer in this producer cell line. Our results show that trichostatin $\mathrm{A}$ induced a higher viral titer than sodium butyrate over a range of concentrations. We then used trichostatin A-treated producer cells to infect primary rat marrow stromal cells (rMSC) and found a significant increase in the number of infected 
cells compared to control infection without any noticeable toxicity. The use of trichostatin A therefore provides a rapid and simple way for producing the increase in viral titer of retroviral producer cell lines, which is useful for any retroviral infection and essential for effective gene therapy protocols.

\section{MATERIALS AND METHODS}

\section{Vectors and Cell Lines}

The nerve growth factor (NGF) retroviral vector was constructed by cloning the NGF mouse cDNA into the Not $\mathrm{I} /$ SnaBI-digested pLIG retroviral vector (provided by Dr. L. Lillien, University of Pittsburgh). The pLIG vector contains the internal ribosomal entry site (IRES) from encephalomyocarditis virus (EMCV) and a reporter/drug resistance gene $(G E O)$ that consists of the bacterial LacZ gene fused with the neomycin phosphotransferase gene. The recombinant retrovirus construct is under the control of the Rous sarcoma virus (RSV) long terminal repeat (LTR) promoter/enhancer elements and is transcribed as a bicistronic mRNA (Figure 1). The retroviral vector DNA was transfected into the ecotropic producer cell line $\mathrm{y}^{2}(12,14)$ using LIPOFECT AMINEÔ (Life Technologies, Rockville, MD, USA). Neomycin-resistant clones were selected using $600 \mathrm{mg} / \mathrm{mL}$ G418 (Life Technologies), and the resulting cell line was designated $y^{2} /$ LIG/NGF. Clones were analyzed for production of virus by infection of NIH 3T3 cells (ATCC, Manassas, VA, USA). rMSC were provided by Dr. Darwin Prockop (MCP Hahnemann University). The harvesting and culturing of the rMSC have been described previously (17).

\section{Trichostatin A and Sodium Butyrate Treatment of $y^{2} /$ LIG/NGF Cells}

Trichostatin A (Sigma, St. Louis, MO, USA) was prepared in ethanol at a concentration of $3 \mathrm{mM}$ and stored at $-20^{\circ} \mathrm{C}$. N-Butyric acid (Sigma) was prepared in distilled water at a concentration of $2.5 \mathrm{M}$ and stored at $-20^{\circ} \mathrm{C}$.

$\mathrm{y}^{2} / \mathrm{LIG} / \mathrm{NGF}$ and NIH $3 \mathrm{~T} 3$ cells were cultured with high glucose DMEM (Life Technologies) containing
$10 \%$ calf serum and antibiotics (penicillin/streptomycin; Life Technologies) at $37^{\circ} \mathrm{C}$ and $5 \% \mathrm{CO}_{2}$. rMSC were cultured under the same conditions but with $10 \%$ fetal calf serum. Retroviral infections were carried out using $\mathrm{y}^{2} /$ LIG/NGF producer cells grown to $80 \%$ confluency in 10-cm plates (Becton Dickinson, Franklin Lakes, NJ, USA). The media was then replaced with either $3 \mathrm{~mL}$ fresh media, fresh media containing trichostatin A or fresh media containing sodium butyrate and incubated for $24 \mathrm{~h}$. NIH $3 \mathrm{~T} 3$ cells or rMSC were plated at a density of 2 ' $10^{5}$ cells per well in a 6-well dish (Becton Dickinson) and grown for $24 \mathrm{~h}$ to approximately $50 \%$ confluency.

Supernatants from producer cells were filtered through a $0.2-\mathrm{mm}$ filter, and the NIH 3 T3 cells or rMSC were infected in the presence of $8 \mathrm{mg} / \mathrm{mL}$ polybrene for $4 \mathrm{~h}$. In some conditions, trichostatin A was only added at the time of infection to serve as a control. The media was then replaced with fresh media, and the NIH 3 T3 cells or rMSC were cultured for $48 \mathrm{~h}$, then processed for X-Gal staining (11) for visualization of the reporter gene b-galactosidase (b-gal) to determine the titer of the virus in the supernatant.

\section{Determination of Viral Titer}

We defined the viral titer as the number of NIH 3T3 cells infected by a virus in a known volume of viral supernatant. In this study, the expression of the $L a c Z$ reporter gene was used as a marker for infected cells. The viral titer was calculated from the number of $b$ gal-positive NIH 3T3 cells infected per milliliter of viral supernatant.

To calculate the number of infected cells by a volume of producer cell su- pernatant, a grid was created to divide a single well of a 6 -well plate into 45 units (Figure 2). Ten units were randomly selected from the 45-unit well and the number of b-gal-positive cells were counted in three nonoverlapping reticule fields contained within each of these units using a $100^{\prime}$ objective with a micrometer. The total number of positive cells in the well was calculated based on the reticule area relative to the entire well (1:1230). These experiments were done in duplicate wells. Therefore, we made 60 separate measurements for each experimental condition (three nonoverlapping fields multiplied by 10 random units done in duplicate wells) to derive the mean number of b-gal-positive cells.

Since the wells were infected in duplicate, one set of $30 \mathrm{~b}$-gal-positive cell counts was compared to the other well's b-gal cell counts by using a paired students $t$-test. The results of the showed that there was no statistical difference between the number of b-galpositive cell counts in one well compared to the duplicate well for the same treatment (data not shown). This allowed counts from duplicate wells to be used to derive the mean number of NIH 3T3 cells or rMSC infected for each experimental condition.

\section{RESULTS AND DISCUSSION}

\section{Trichostatin A Increases the Viral Titer of $y^{2} / \mathrm{LIG} / \mathrm{NGF}$ Cells}

Five concentrations (3.0, 1.0, 0.5, 0.25 and $0.1 \mathrm{mM}$ ) of trichostatin $\mathrm{A}$ were used to treat the $\mathrm{y}^{2} / \mathrm{LIG} / \mathrm{NGF}$ cells. One-way between subjects analysis of variance (ANOVA) showed that trichostatin A increased the viral titer of

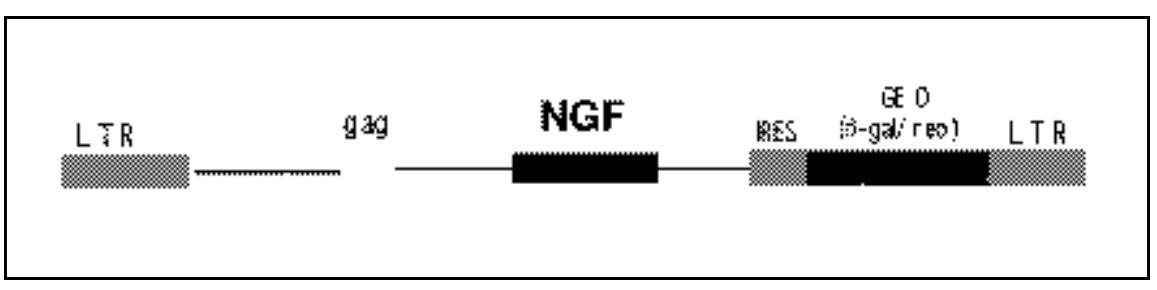

Figure 1. The structure of the LIG/NGF retrovirus. The virus encodes the mouse NGF transgene and $G E O$, which is a fusion of the b-gal and neomycin phosphotransferase genes, allowing the visual identification of infected cells (b-gal) as well as selection of cells by G418. The recombinant construct is driven by the RSV LTR promoter/enhancer elements. The IRES sequence located between NGF and GEO allows the translation of the polycistronic mRNA. 
$\mathrm{y}^{2} / \mathrm{LIG} / \mathrm{NGF}$ producer cells in a dosedependent manner, $\mathrm{F}(6,133)=43.5, P$ $<0.0001$ (Figure 3). The highest concentration of trichostatin $\mathrm{A}$ in our experiments was $3 \mathrm{mM}$ because this was the concentration that previously produced the maximal activation of viral gene expression (2), and at higher concentrations we observed considerable cell death. The mean number of NIH 3T3 cells infected with supernatant from control untreated producer cells was 5006. Treatment with 3.0, 1.0, 0.5, 0.25 and $0.1 \mathrm{mM}$ trichostatin $\mathrm{A}$ increased the mean viral titer by 34-, 19.2-, 15.3-, 6.9- and 3-fold, respectively. The increase in viral titer by trichostatin A was statistically significant $(P<0.05)$ at the $0.25-\mathrm{mM}$ concentration and highly significant $(P<0.001)$ at the higher concentrations when com pared to the control infection as indicated by the Fisher's least significant difference (LSD) test (Figure 3). The increase in the viral titer by trichostatin A is also shown in Figure 5, where the $\mathrm{X}$-Gal staining is presented.

These results represent a novel application of trichostatin A for increasing titers of retroviruses by using a simple and effective protocol. The $y^{2} / \mathrm{LIG} / \mathrm{NGF}$ producer clone used in this study had a very low viral titer $\left(5^{\prime} 10^{3}\right)$ that would have severely reduced its usefulness. Treatment with trichostatin A increased the viral titer of a producer cell line sufficiently $\left(>10^{5}\right)$ to make it useful.

We performed two additional experiments to determine if trichostatin A's effect on viral titer resulted from an action on the producer cells (producing an increased total viral particle number) or the target cells (producing an increased susceptibility to infection) and to determine if trichostatin A had similar effects on a primary cell line. To answer the first question, we applied 3 $\mathrm{mM}$ trichostatin A (optimal concentration determined in our experiments) only at the time of infection of NIH 3T3 cells or primary rMSC and not during the 24-h incubation period with the $y^{2} /$ LIG/NGF producer cells. As observed in the previous experiment, ANOVA showed that $3 \mathrm{mM}$ trichostatin A applied to the producer cells increased the viral titer upon infection of NIH 3 T3 cells, $\mathrm{F}(2,57)=1694.4, P<$ 0.0001 (Figure 4). Treatment with 3
$\mathrm{mM}$ trichostatin A increased the mean viral titer 34-fold compared to the control and was highly statistically significant $(P<0.001$, Fischer's LSD test). In contrast, trichostatin A administration only at the time of infection of $\mathrm{NIH}$ $3 \mathrm{~T} 3$ cells did not significantly increase the viral titer $(P>0.05$, Fisher's LSD test, Figure 4). This result shows that trichostatin A acts on the producer cells

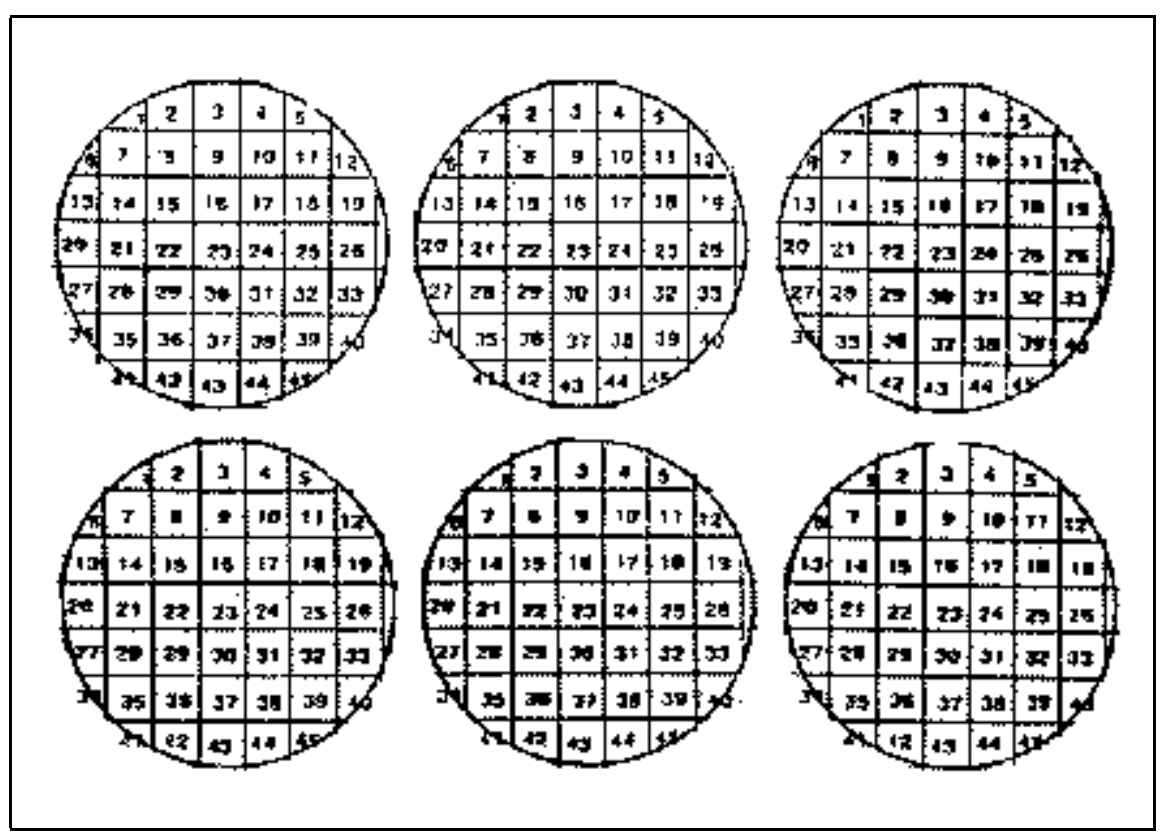

Figure 2. Diagram of the 45 -unit grid used to separate each well of a 6 -well plate into 45 units. The grid was placed under a 6-well plate, and the b-gal-positive cells were counted from three nonoverlapping fields of the randomly generated unit under a $100^{\prime}$ objective.

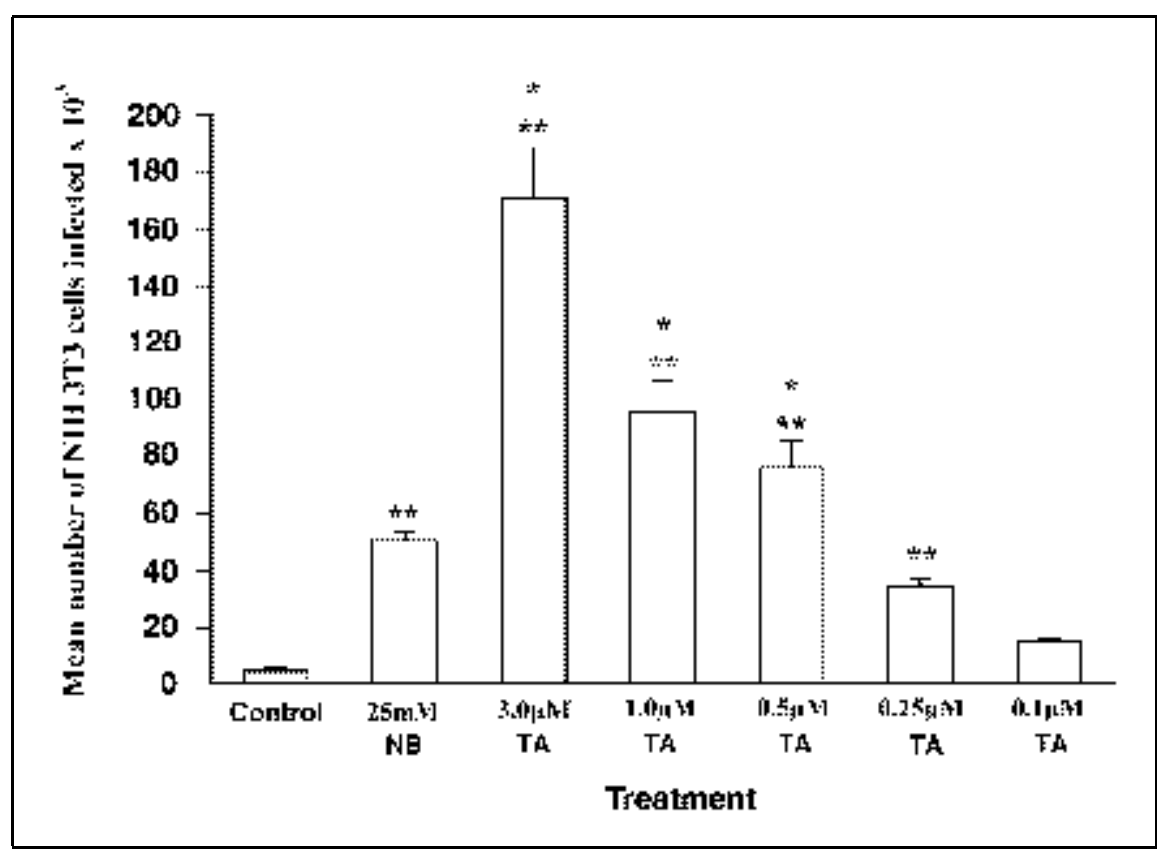

Figure 3. Bar graph comparing viral titers obtained with trichostatin $A$ and sodium butyrate to control treatment and viral titers of trichostatin A to sodium butyrate treatment. Trichostatin A ( $3 \mathrm{mM})$ produced a 34-fold increase in viral titer over control infection. Sodium butyrate $(25 \mathrm{mM})$ caused a tenfold increase in titer over control infection. TA, trichostatin A; NB, sodium butyrate; error bars, \pm SEM; ${ }^{*}$, significantly different values from the control; *, significantly different values from NB. 
to produce the increase in viral titer and not on the NIH $3 \mathrm{~T} 3$ cells.

To answer the second question, we investigated whether $3 \mathrm{mM}$ trichostatin A could increase the number of rMSC cells infected by the $y^{2} /$ LIG/NGF producer cells. ANOVA showed a highly significant increase in the number of infected rMSC, $\mathrm{F}(2,57)=857.1, P<$ 0.0001 (Figure 4). Treatment increased the number of rMSC infected 5.8-fold compared to control infection and was highly statistically significant $(P<$ 0.001 , Fisher's LSD test) without visible toxicity. When $3 \mathrm{mM}$ trichostatin A was added only at the time of infection, there was no significant increase in the number of infected rMSC compared to control infection $(P>0.05$, Fisher's LSD test, Figure 4). This result shows that trichostatin $\mathrm{A}$ is an effective agent for increasing infection of a primary cell line (rMSC) that has been shown to be useful for ex vivo gene therapy in a rat model of Parkinson's disease (17). In previous studies, trichostatin $\mathrm{A}$ has also been shown not to be toxic to hematopoietic clonal cell lines (13) and human intestinal epithelial cells (19) growing in culture. Long-term exposure to trichostatin A may put some cells into growth arrest $(19,20)$, but the limited exposure ( $4 \mathrm{~h}$ during infection) required by this method is unlikely to cause a considerable amount of cell death in the target cell line. The decrease in viral titer utilizing $3 \mathrm{mM}$ trichostatin A with rMSC compared to $\mathrm{NIH} 3 \mathrm{~T} 3$ cells is consistent with the frequently reported finding that primary cells lines are more resistant to infection than transformed clonal cell lines such as NIH 3T3 cells.

\section{Trichostatin A Is More Effective than Sodium Butyrate}

We treated the $\mathrm{y}^{2} / \mathrm{LIG} / \mathrm{NGF}$ producer cells with a range of concentrations of sodium butyrate that are known to increase viral titers $(0.5-50 \mathrm{mM})$ and found that $25 \mathrm{mM}$ sodium butyrate provided the highest increase in viral titer in the NIH 3 T3 cell assay. At $25 \mathrm{mM}$, the mean viral titer was 50755 . This concentration of sodium butyrate therefore produced a tenfold increase over control, which was statistically signifi-

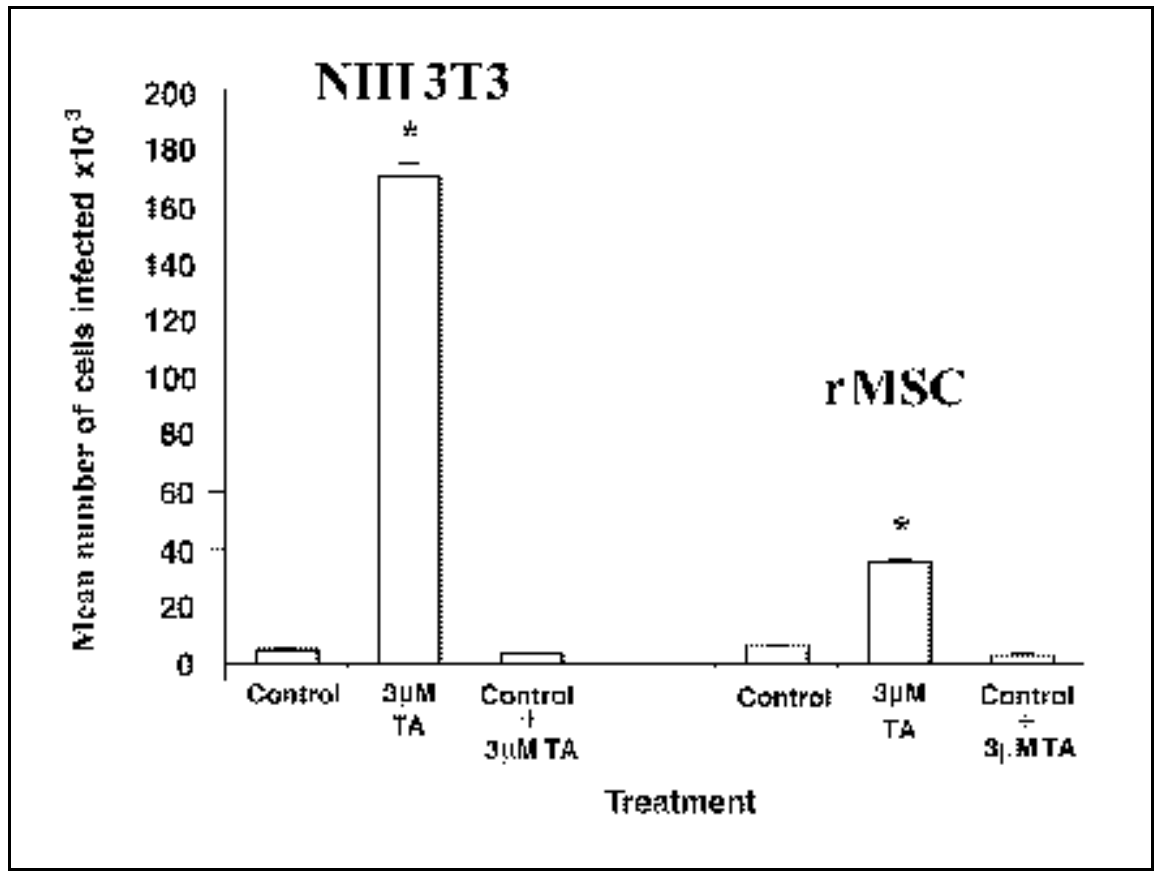

Figure 4. Bar graph comparing viral titers obtained with control treatment, $3 \mathrm{mM}$ trichostatin A applied to producer cells or when $3 \mathrm{mM}$ trichostatin $\mathrm{A}$ is applied only at the time of infection on NIH 3T3 cells or rMSC. Trichostatin A $(3 \mathrm{mM})$ produced a 34-fold increase in viral titer over control infection in NIH 3T3 cells and a 5.8-fold increase in rMSC. This increase was abolished when $3 \mathrm{mM}$ trichostatin A was applied only during infection of NIH $3 \mathrm{~T} 3$ cells or rMSC (control plus $3 \mathrm{mM} \mathrm{TA}$ ). TA, trichostatin A; error bars, $\pm \mathrm{SEM}$ *, significantly different values from the controls. 


\section{Research Report}

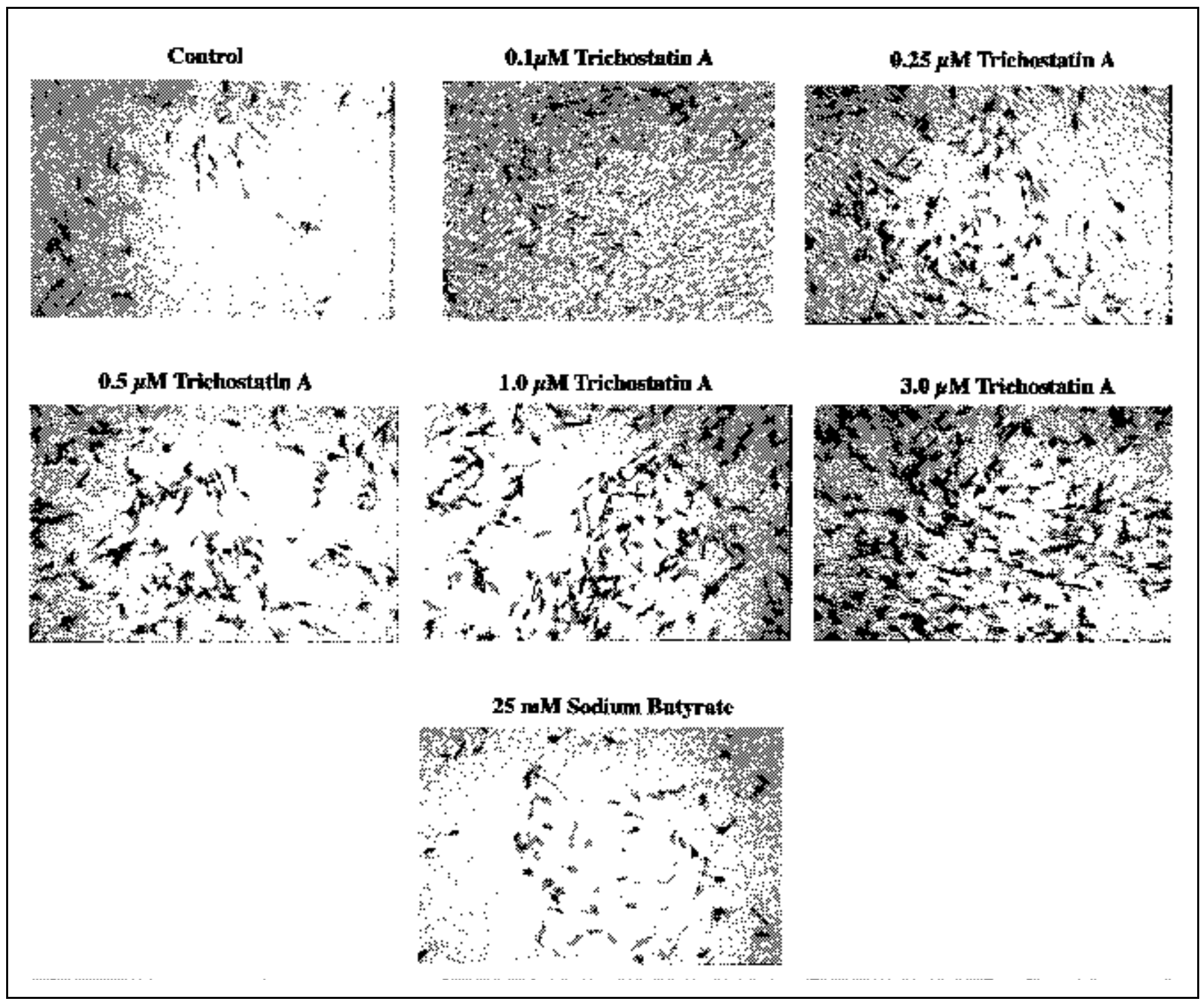

Figure 5. Photomicrographs of representative b-gal staining of NIH 3T3 cells after trichostatin A, sodium butyrate or control treatments under $100^{\circ}$ magnification. Note the largest increase in b-gal-positive cells with $3 \mathrm{mM}$ trichostatin $\mathrm{A}$.

cant $(P<0.001$, Fisher's LSD test, Figure 3). At concentrations of 3.0, $1.0(P$ $<0.001)$ and $0.5 \mathrm{mM}(P<0.05)$, trichostatin A produced viral titers that were significantly greater than those attained with $25 \mathrm{mM}$ sodium butyrate (Figure 3 ). The titer produced by $3 \mathrm{mM}$ trichostatin A was 3.4-fold higher than that produced by $25 \mathrm{mM}$ sodium butyrate and raised the mean number of cells infected from 50755 to 170460 .

These results are in agreement with previous studies of trichostatin A and sodium butyrate, which showed that trichostatin A had more potent effects at lower concentrations than sodium bu- tyrate $(3,4)$. The mechanism of trichostatin A's actions is still not completely understood but depends on the drug's ability to inhibit the enzyme histone deacetylase. This results in the relaxation of genomic DNA, which can increase retroviral gene expression (3). Inhibitors of histone deacetylase may also have inhibitory effects on the cell cycle $(18,19)$, which may also increase production of viral particles.

This study demonstrates the effectiveness of an inhibitor of histone deacetylase, trichostatin A, in increasing the retroviral titer of the producer cell line $y^{2} /$ LIG/NGF. Trichostatin A offers the ability to increase the retroviral titer of this producer cell line 34-fold over the control infection and 3.4-fold higher than the commonly used compound sodium butyrate. Ex vivo gene therapy's efficiency is critically dependent on the ability of the virus to infect as many cells as possible in vitro so that the cells can deliver the highest possible amount of therapeutic transgene when they are introduced in vivo. We found that trichostatin A increased the number of infected rMSC 5.8-fold over the control infection. This increase in the number of infected cells could be the difference in eliciting a therapeutic effect when the 
cells are grafted back into the host.

In contrast to the elaborate and timeconsuming procedure that is involved in creating new producer clones (6), the method described here is simple and effective. Trichostatin A is easy to obtain and store, and the procedure is subject to virtually no time constraints when used in a retroviral infection experiment. Because sodium butyrate can elevate some high-titer clones $\left(10^{5}\right)$ even higher $\left(10^{6}\right)(15)$, it would be worth examining whether trichostatin A has similar effects on a high-titer clone. Trichostatin A might also be combined with other strategies to produce a further increase in viral titer, for example, using a lower temperature during retroviral harvest $(7,8)$ and/or using low serum (5) along with trichostatin A. Trichostatin A may therefore potentially be used as a simple protocol to increase the viral titer of any type of retrovirus used in ex vivo gene therapy.

\section{ACKNOWLEDGMENTS}

This work was supported by National Institutes of Health grant no. 24707, the Christopher Reeve Research Foundation and MCP Hahnemann University. We thank Dr. L. Lillien for the generous gift of the LIG retrovirus vector, Dr. D. Prockop for the gift of the primary rat marrow stromal cells and Dr. J. Solowska-Baird for constructing LIG/ NGF. We thank Drs. M. Murray and A. Tessler for their critical review of the manuscript and Drs. S.Y. Chow, Y. Liu and Ms. L. Hodge for invaluable advice and technical help.

\section{REFERENCES}

1.Candido, E.P., R. Reeves and J.R. Davie. 1978. Sodium butyrate inhibits histone deacetylation in cultured cells. Cell 1:105-113.

2.Chen, W.Y., E.C. Bailey, S.L. McCune, J.-Y. Dong and T.M. Townes. 1997. Reactivation of silenced, virally transduced genes by inhibitors of histone deacetylase. Proc. Natl. Acad. Sci. USA 94:5798-5803.

3.Chen, W.Y. and T.M. Townes. 1999. Molecular mechanisms for silencing virally transduced genes involves histone deacetylation and chromatin condensation. Proc. Natl. Acad. Sci. USA 97:377-382.

4.Condreay, J.P., S.M. Witherspoon, W.C. Clay and T.A. Kost. 1999. Transient and stable gene expression in mammalian cells transduced with a recombinant baculovirus vector. Proc. Natl. Acad. Sci. USA 96:127-132.

5.Glimm, H., K. Flugge, D. Mobest, V.M. Hofmann, J. Postmus, R. Henschler, W. Lange, J. Finke et al. 1998. Efficient serum-free retroviral gene transfer into primitive human hematopoietic progenitor cells by a defined, high-titer, nonconcentrated vector-containing medium. Hum. Gene Ther. 6:771-778.

6.Hwang, L.H. and E. Gilboa. 1984. Expression of genes introduced into cells by retroviral infection is more efficient than that of genes introduced into cells by DNA transfection. J. Virol. 50:417-424.

7.Kaptein, L.C., A.E. Greijer, D. Valerio and V.W. Beusechem. 1997. Optimized conditions for the production of recombinant am photropic retroviral vector preparations. Gene Ther. 2:72-76.

8.Kotani, H., P.B. Newton III, S. Zhang, Y.L. Chiang, E. Otto, L. Weaver, R.M. Blaese, W.F. Anderson and G.J. McCarrity. 1994. Improved methods of retroviral vector transduction and production for gene therapy. Hum. Gene Ther. 1:19-28.

9.Kruh, J. 1982. Effects of sodium butyrate, a new pharmacological agent, on cells in culture. Mol. Cell Biochem. 42:65-82.

10.Lee, S.G., S. Kim, P.D. Robbins and B.G. Kim. 1996. Optimization of environmental factors for the production and handling of re- combinant retrovirus. Appl. Microbiol. Biotechnol. 45:477-483.

11.Liu, Y., B.T. Himes, J. Moul, W.L. Huang, S.Y. Chow, A. Tessler and I. Fischer. 1997. Application of recombinant adenovirus for in vivo gene delivery to spinal cord. Brain Res. 768:19-29.

12.Mann, R., R.C. Mulligan and D. Baltimore. 1983. Construction of a retrovirus packaging mutant and its use to produce helper-free defective retrovirus. Cell 33:153-159.

13.McInerney, J.M., J.R. Nawrocki and C.H. Lowrey. 2000. Long-term silencing of retroviral vectors is resistant to reversal by trichostatin A and 5-azacytidine. Gene Ther. 7:653663.

14.Miller, AD. 1990. Retrovirus packaging cells. Hum. Gene Ther. 1:5-14.

15.Olsen, J.C. and J. Sechelski. 1995. Use of sodium butyrate to enhance production of retroviral vectors expressing CFTR cDNA. Hum. Gene Ther. 5:1195-1202.

16.Paul, R.W., D. Morris, B.W. Hess, J. Dunn and R.W. Overell. 1993. Increased viral titer through concentration of viral harvests from retroviral packaging lines. Hum. Gene Ther. 5:609-615.

17.Schwarz, E.J., G.M. Alexander, D.J. Prockop and S.A. Azizi. 1999. Multipotential marrow stromal cells transduced to produce LDopa: engraftment in a rat model of parkinson disease. Hum. Gene Ther. 10:2539-2549.

18.Shadan, F.F., L.M. Cowsert and L.P. Villarreal. 1994. n-Butyrate, a cell cycle blocker, inhibits the replication of polyomaviruses and papillomaviruses but not that of adenoviruses and herpesviruses. J. Virol. 68:4785-4796.

19.Siavoshian, S., J.-P. Segain, M. Kornprobst, C. Bonnet, C. Cherbut, J.-P. Galmiche and H.M. Blottiere. 2000. Butyrate and trichostatin A effects on the proliferation/differentiation of human intestinal epithelial cells: induction of cyclin D3 and p21 expression. Gut 46:507-514.

20.Yoshida, M. and T. Beppu. 1988. Reversible arrest of proliferation of rat $3 \mathrm{Y} 1$ fibroblasts in both the G1 and G2 phases by trichostatin A. Exp. Cell Res. 177:122-131.

21.Yoshida, M., M. Kijima, M. Akita and T. Beppu. 1990. Potent and specific inhibition of mammalian histone deacetylase both in vivo and in vitro by trichostatin A. J. Biol. Chem. 265:17174-17179.

22.Yoshida, M., S. Horinouchi and T. Beppu. 1995. Trichostatin A and trapoxin: novel chemical probes for the role of histone acetylation in chromatin structure and function. Bioessays 5:423-430.

Received 22 February 2000; accepted 29 June 2000.

Address correspondence to:

Dr. Itzhak Fischer

Department of Neurobiology and Anatomy

MCP Hahnemann University

2900 Queen Lane

Philadelphia, PA 19129, USA

e-mail: itzhak.fischer@drexel.edu 